\title{
Small Cell Carcinoma of the Ovary: Report of Three Cases of a Poor Prognosis Disease
}

\author{
Massimo Origonia, b, Alice Bergamini ${ }^{\mathrm{a}}$, Francesca Pella ${ }^{\mathrm{a}}$, Jessica Ottolina ${ }^{\mathrm{a}}$, \\ Veronica Giorgione ${ }^{a}$, Diana Del Prato ${ }^{a}$, Giada Almirante ${ }^{a}$, \\ Massimo Candiani ${ }^{\mathrm{a}}$
}

\begin{abstract}
Small cell carcinoma of the ovary (SCCO) is a rare, malignant and very aggressive tumor. According to the current state of the art, no standard treatment has been established, both in terms of treatment modalities and outcomes. With the exception of some very rare cases reported in literature, the prognosis remains very poor and mortality rate extremely high. We report of three cases of SCCO with rapid progression and spreading of the disease despite multifactorial therapies. Different treatment options have been administered to our patients, but none of them was successful in achieving satisfactory outcomes and/or disease control.
\end{abstract}

Keywords: Ovarian cancer; Small cell; SCCO; Chemotherapy; Hypercalcemia

\section{Introduction}

Small cell carcinoma of the ovary (SCCO) is a malignant and highly aggressive tumor, which tends to progress and spread very fast despite therapy. It is associated with a poor prognosis and a high mortality rate. This type of tumor was first described in details by Dickersin and Scully in the 1980s [1]. It mostly occurs in young women and adolescents, with a mean age of 23 yrs. (range 1 - 46) [1] at diagnosis. About $50 \%$ of patients presents with an advanced stage of disease at the moment of diagnosis [2]. Two different histological vari-

Manuscript accepted for publication November 20, 2012

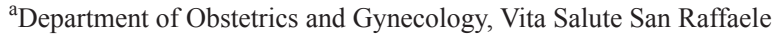
University School of Medicine, Milano, Italy

${ }^{\mathrm{b}}$ Corresponding author: Massimo Origoni, Department of Obstetrics and Ginecology - Vita Salute San Raffaele University School of Medicine, San Raffaele Scientific Institute, Via Olgettina 60, 20132 Milano, Italy. Email: massimo.origoni@hsr.it

doi: http://dx.doi.org/10.4021/jmc1043e eties of SCCO have been described: one similar to small cell carcinoma of the lung, the other, more uncommon and less known, characterized by the presence of a large cell component (large cell variant of SCCO) [3]. Approximately $60 \%$ of SCCO are associated with paraneoplastic hypercalcemia [1]. Tendency to progression and recurrence are the main features of this malignancy. Many series published throughout the years highlight the poor prognosis of this tumor, despite the different treatment modalities applied. Many different therapeutical options have been considered in the treatment of SCCO, but nevertheless the outcome remains very poor and the mortality rate extremely high. A limited number of published studies are available, mainly represented by case reports [1,4-7]. We report of three cases of SCCO with an extremely rapid progression and spreading of the disease, with ineffectiveness of the different treatment approaches utilized.

\section{Case Report}

\section{Case 1}

A 16-year-old girl was admitted to the Emergency Unit of our institution for symptoms suggesting bowel occlusion. Two weeks before she had undergone a left salpingo-oophorectomy, omentectomy and multiple abdomino-pelvic biopsies, with the suspicion of a monolateral malignant ovarian germ cell tumor. Computed tomography (TC) scan revealed a massive, solid, abdomino-pelvic progression of disease. Therefore she underwent secondary surgery: a large neoplastic pelvic solid mass was detected, and a total abdominal hysterectomy with controlateral salpingo-oophorectomy, rectal and left large bowel nodules excisions were performed, along with omental, sigmoid and prevescical peritoneum multiple biopsies. Several neoplastic nodules of $1-2 \mathrm{~cm}$. size in the abdominal peritoneum and diaphragmatic surface were left unresected. Microscopic examination of the surgical specimens revealed metastatic involvement from SCCO (stage IIIC G3, according to FIGO classification of gynaecological malignancies). Few days later a further abdominopelvic progression of disease was clinically observed and 
a diffuse internal mammary lymphadenopathy completely involving the sternum occurred. General conditions of the patients rapidly worsened, excluding the chance of an early adjuvant therapy. The patient died for disease progression five weeks after the first surgery.

\section{Case 2}

A 34-year-old patient presented with ascites, right pleural effusion and a right ovarian mass. Computed tomography scan revealed a $13 \mathrm{~cm}$ right, solid adnexal mass; serum CA 125 was $121 \mathrm{UI} / \mathrm{mL}$, Bhcg $17 \mathrm{U} / \mathrm{L}$, calcemia $4.1 \mathrm{mmol} / \mathrm{L}$.

She underwent right salpingo-oophorectomy, pelvic and para-aortic lymphadenectomy, total omentectomy. Frozensection histological examination of the surgical specimen revealed an undifferentiated neoplasia suggestive of ovarian dysgerminoma. Through definitive histopathology report the diagnosis of SCCO (FIGO Stage III C) was made. Ten days after surgery she developed bowel obstruction. Computed tomography imaging documented massive abdomino-pelvic and lymphatic progression. At second laparotomy, uterus appeared completely infiltrated by the tumor, without surgical cleavage from the bladder; a left oophorectomy and ileo-transverse side to side anastomosis were performed, secondary to large bowel resection. Histological examination confirmed SCCO progression. Two weeks after surgery, adjuvant poli-chemotherapy was started according to VPBCAE regimen (vinblastin $6 \mathrm{mg} / \mathrm{mq}$ day 1 , cisplatinum $90 \mathrm{mg} / \mathrm{mq}$ day 1 , bleomycin $15 \mathrm{mg} / \mathrm{mq}$ day 2 , cyclophosphamide $750 \mathrm{mg} / \mathrm{mq}$ day 2 , doxorubicin $45 \mathrm{mg} / \mathrm{mq}$ day 3 , etoposide $200 \mathrm{mg} / \mathrm{mq}$ day 3, administered every 21 days). After two consecutive courses of chemotherapy a CT scan revealed massive abdomino-pelvic progression of disease. The patient soon developed bowel occlusion and died five months after diagnosis.

\section{Case 3}

A 53-year-old woman presented with a $19 \times 10 \times 9 \mathrm{~cm}$ biloculated solid ovarian cyst. Serum CA125 was $343 \mathrm{UI} / \mathrm{mL}$. Preoperative calcium level was $3.32 \mathrm{mmol} / \mathrm{L}$ and LDH 696 $\mathrm{UI} / \mathrm{L}$.

She underwent a total abdominal hysterectomy, bilateral salpingo-oophorectomy, and total omentectomy. A terminal ileum resection with ileo-colic side to side anastomosis was necessary due to intraoperative ileum perforation. A residual $5 \mathrm{~cm}$ neoplastic nodule, close to right internal iliac vessels, was left unresected. Histological diagnosis was of SCCO of the hypercalcemic type FIGO Stage IIIC. Three weeks after surgery, patient complained of fever and malaise. A CT scan revealed marked progression of disease: a $3 \times 2.5 \times 3.3 \mathrm{~cm}$ right IV costal metastasis associated with rib fracture was detected and several other solid nodules were also documented in both lungs, mediastinal and ascella lymphnodes, adrenal gland, pelvis, iliac and para-aortic lymphnodes and on the ileocolic serosa. Ten days later, the first course of adjuvant poli-chemotherapy regimen with PAVEP (cisplatin (P) 80 $\mathrm{mg} / \mathrm{mL}$ day 1 , adriamycin (A) $40 \mathrm{mg} / \mathrm{mq}$ day 1 , vepeside (V) $75 \mathrm{mg} / \mathrm{mq} /$ day days 1 - 3, cyclophosphamide (EP) $300 \mathrm{mg} /$ mq/day days 1 - 3 every 3 weeks) was administered. A total of three adjuvant chemotherapy courses were administered. CT evaluation performed two months after initiation of chemotherapy revealed a marked reduction of lungs nodules, a complete regression of adrenal mass but an enlargement of the costal metastasis and pelvic mass. One month later, 1 course of high-dose chemotherapy was started, consisting of carboplatin (CARBOPEC regimen: $400 \mathrm{mg} / \mathrm{mq} /$ day days 1 - 4), vepeside (450 mg/mq/day days 1 - 4) and cyclophosphamide $(1,600 \mathrm{mg} / \mathrm{mq} /$ day days 1 - 4). CT scan, performed after high dose chemotherapy, documented enlargement of costal metastasis $(7 \mathrm{~cm})$, several liver nodules, enlargement of pelvic mass $(11 \times 11 \times 9 \mathrm{~cm})$, peritoneal carcinosis and ascites. The patient soon developed bowel obstruction and died few days later, six months after diagnosis.

\section{Discussion}

SCCO is an undifferentiated neoplasm; actually, whether the origin of tumor cells is epithelial, germinal or mesenchymatous (the cell lineage remains not clearly determined) [8, 9] it is not known. The differential diagnosis includes sex cord tumors, dysgerminoma, ovarian metastasis of lymphoma, alveolar rhabdomyosarcoma or melanoma [10]. Moreover, there are two different varieties of small cell carcinoma of the ovary: one is similar to small cell carcinoma of the lung and the other, more uncommon and less known, includes a large cells component (large cell variant of SCCO) [3]. Approximately $60 \%$ of SCCO are associated with paraneoplastic hypercalcemia that often leads the patient in seeking medical attention for nausea, vomiting, abdominal pain, constipation, lethargy, weakness, confusion; despite these symptoms, clinical manifestations of hypercalcemia are rarely reported [1]. SCCO is a prognostically very unfavorable disease. Although it seems to be both chemo and radiosensitive, leading to a high initial response rate, the disease progression-free survival is usually very short, with a median survival of 6 - 13 months (range of 2 - 23 months) [5], with the exception of early stages disease: in the largest published series in literature, only $33 \%$ of the patients with stage IA are alive and free of disease [4]. Tendency to progression and recurrences are the two main features of this malignancy. Many published series throughout the years appear to highlight the poor prognosis of this tumor; more than $50 \%$ of patients are diagnosed with advanced stages disease (stage III or higher) [2]. Approximately $30 \%$ of patients staged IA had a long recurrence-free survival compared to $10 \%$ of cases staged IC and $6.5 \%$ of women in advanced stage, respectively. As 
a matter of fact, when the tumor has extrapelvic spreading, survival for more than few years is reported to be almost exceptional [1].

Several different chemotherapy treatment options have been advocated for SCCO patients [6]. Nevertheless, the prognosis generally remains dramatically poor. Few large studies have been conducted [1, 5-7], the more significant one by Young [4]. Adjuvant regimens were derived from epithelial carcinoma therapies, consisting of cisplatinum, cyclophosphamide with or without doxorubicine and hexamethylmelamine; in all cases only short-term favourable responses have been obtained [5]. Paclitaxel or platinum therapy in advanced stage disease had no or little efficacy either. Longterm survivors were reported only in clinical studies using a multiagent chemotherapy approach, consisting of regimens alternating cycles with vinblastine, cisplatin, bleomycin (PVB) and cyclophosphamide, doxorubicin, and etoposide with or without isophosphamide [11], or adjuvant induction therapy with PEB (the same used in germ cells tumors) followed by consolidation cycles of vincristine, actinomycin D, and cyclophosphamide (VAC) [12]. Another limited number of long-term survivors with advanced stages disease at diagnosis had received regimens consisting of 5 to 6 antineoplastic agents (combination of vinblastine, cisplatin, cyclophosphamide, bleomycin, adriamycin, etoposide) [8, 9]. Other series, the most recent of which by Pautier in 2007, describe poli-chemotherapy as first line treatment in SCCO of the hypercalcemic type followed by high dose consolidation chemotherapy leading to an exceptionally better outcome $[6,7]$. In our experience, this therapeutic attempt was unsuccessful. The role of radiotherapy has not yet been definitely established because of the limited number of cases in literature; it has been indicated as useful in the multimodality treatment of SCCO as advocated by Harrisons in 2006 [5]. From the literature review, currently no standard treatment for SCCO can be advocated. This depends both on the rarity of the disease and the lack of randomized controlled trials ever been performed; most of the clinical studies published are small series or case reports. In most cases the progression and spread of this disease is so fast that patients cannot receive chemotherapy or are not eligible for surgery due to terminal general conditions. Since there is no consensus on the treatment of SCCO and no success of standard therapies has been highlighted in literature, other molecules potentially effective on SCCO cells should be investigated in clinical, multi-centre studies, although the rarity, rapid spreading and poor prognosis of the disease represent objective obstacles gynaecological oncologists have to face with, in the challenge of increasing survival rates for these patients.

\section{Conflict of Interest}

Authors declare that no conflict of interest has to be dis- closed.

\section{Financial Support}

Authors declare that no financial support has been obtained for the preparation of the manuscript.

\section{References}

1. Dickersin GR, Kline IW, Scully RE. Small cell carcinoma of the ovary with hypercalcemia: a report of eleven cases. Cancer. 1982;49(1):188-197.

2. Patsner B, Piver MS, Lele SB, Tsukada Y, Bielat K, Castillo NB. Small cell carcinoma of the ovary: a rapidly lethal tumor occurring in the young. Gynecol Oncol. 1985;22(2):233-239.

3. Baeyens L, Amat S, Vanden Houte K, Vanhoutte P, L'Hermite M. Small cell carcinoma of the ovary successfully treated with radiotherapy only after surgery: case report. Eur J Gynaecol Oncol. 2008;29(5):535-537.

4. Young RH, Oliva E, Scully RE. Small cell carcinoma of the ovary, hypercalcemic type. A clinicopathological analysis of 150 cases. Am J Surg Pathol. 1994;18(11):1102-1116.

5. Harrison ML, Hoskins $\mathrm{P}$, du Bois A, Quinn M, Rustin GJ, Ledermann JA, Baron-Hay S, et al. Small cell of the ovary, hypercalcemic type -- analysis of combined experience and recommendation for management. A GCIG study. Gynecol Oncol. 2006;100(2):233-238.

6. Distelmaier F, Calaminus G, Harms D, Strater R, Kordes U, Fleischhack G, Gobel U, et al. Ovarian small cell carcinoma of the hypercalcemic type in children and adolescents: a prognostically unfavorable but curable disease. Cancer. 2006;107(9):2298-2306.

7. Pautier P, Ribrag V, Duvillard P, Rey A, Elghissassi I, Sillet-Bach I, Kerbrat P, et al. Results of a prospective dose-intensive regimen in 27 patients with small cell carcinoma of the ovary of the hypercalcemic type. Ann Oncol. 2007;18(12):1985-1989.

8. Senekjian EK, Weiser PA, Talerman A, Herbst AL. Vinblastine, cisplatin, cyclophosphamide, bleomycin, doxorubicin, and etoposide in the treatment of small cell carcinoma of the ovary. Cancer. 1989;64(6):1183-1187.

9. Sholler GL, Luks F, Mangray S, Meech SJ. Advanced small cell carcinoma of the ovary in a pediatric patient with long-term survival and review of the literature. J Pediatr Hematol Oncol. 2005;27(3):169-172.

10. Aguirre P, Thor AD, Scully RE. Ovarian small cell carcinoma. Histogenetic considerations based on immunohistochemical and other findings. Am J Clin Pathol. 1989;92(2):140-149.

11. Tewari K, Brewer C, Cappuccini F, Macri C, Rogers 
LW, Berman ML. Advanced-stage small cell carcinoma of the ovary in pregnancy: long-term survival after surgical debulking and multiagent chemotherapy. Gynecol Oncol. 1997;66(3):531-534.
12. Powell JL, McAfee RD, McCoy RC, Shiro BS. Uterine and ovarian conservation in advanced small cell carcinoma of the ovary. Obstet Gynecol. 1998;91(5 Pt 2):846848. 